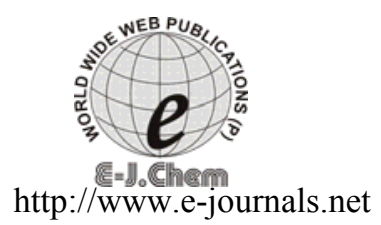

ISSN: 0973-4945; CODEN ECJHAO

E-Journal of Chemistry

2011, 8(S1), S149-S154

\title{
Synthesis and Antimicrobial Screening of Some Novel 2, 5-Disubstituted 1, 3, 4-oxadiazole Derivatives
}

\author{
P. PANNEERSELVAM ${ }^{*}$ and G. GEETE GANESH \\ Department of Pharmaceutical Chemistry \\ C.L.Baid Metha College of Pharmacy \\ Thoraipakkam, Chennai-600 097, Tamilnadu, India \\ pps2k2000@yahoo.co.in
}

Received 14 March 2011; Accepted 30 April 2011

\begin{abstract}
The syntheses of series of 2, 5-disubstituted 1, 3, 4-oxadiazole derivatives are described. A total of twelve new compounds were synthesized and characterized by IR, ${ }^{1} \mathrm{H}-\mathrm{NMR}$ and Mass spectral data. All newly synthesized compounds were screened for their antimicrobial activity i.e. antibacterial activity against $S$. aureus and $E$. coli and antifungal activity against fungus $A$. nigar. Compounds G5 and G7 exhibited significant both antibacterial and antifungal activity while G2, G10 and G3, G9 showed antibacterial and antifungal activity respectively. These compounds were 2 , 5-disubstituted 1, 3, 4-oxadiazole moiety at position two and five showed reasonable antibacterial and antifungal activity.
\end{abstract}

Keywords: 1, 3, 4-Oxadiazole, p-Acetamido phenol, Hydrazine hydrate, Microwave irradiation, Antimicrobial activity

\section{Introduction}

The chemistry of heterocyclic compounds continues to be an active field in the organic chemistry. In the past decades the problem of multidrug resistant microorganism has reached on alarming level around the world, and the synthesis of new antiinfecctive compounds has become an urgent need for the treatment of microbial infections. 1,3,4-Oxadiazole nucleus has been incorporated in to a wide variety of therapeutically important agents mainly displaying antimicrobial ${ }^{1}$, anticonvulsant ${ }^{2}$, anthelmintics ${ }^{3}$, analgesic ${ }^{4}$, insecticidal ${ }^{5}$, local anaesthetic $^{6}$, antidiarrheal ${ }^{7}$, anticancer ${ }^{8}$ and vasorelaxant ${ }^{9}$ activities. In view of these finding and in continuation to our interest in the synthesis and biological activities of 1, 3, 4-oxadiazole,we now report the some novel 2, 5-disubstituted 1, 3, 4-oxadiazole derivatives derived by using $p$-acetamido phenol, ethyl chloroacetate, hydrazine hydrate and phosphorous oxychloride. 


\section{Experimental}

Melting points were determined on a Gallen Kamp apparatus in open capillaries and are uncorrected. IR spectra $\left(\mathrm{KBr}\right.$ in $\left.\mathrm{cm}^{-1}\right)$ were recorded on ABB Bomem FT-IR spectrophotometer and ${ }^{1} \mathrm{H}-\mathrm{NMR}$ spectra (DMSO-d6) on a BRUKAR NMR using TMS as an internal standard (chemical shift in $\delta \mathrm{ppm}$ ). Mass spectra were recorded on JEOL GCmate. The purity of the compounds was confirmed by TLC on precoated silica gel $\left(\mathrm{HF}_{254}, 200\right.$ mesh) aluminium plates (E Merck).

Synthesis of ethyl -4-acetamido phenoxy acetate $(\mathbf{1})^{10}$

A mixture of $p$-acetamido phenol $(0.01 \mathrm{~mol})$ and ethyl chloroacetate $(0.01 \mathrm{~mol})$ was refluxed by using dry acetone in presence of anhydrous potassium carbonate $\left(\mathrm{K}_{2} \mathrm{CO}_{3}\right)$ for $6 \mathrm{~h}$. The reaction mixture was cooled and then poured in to crushed ice. The solid so product obtained was filtered, dried and recrystallized using ethanol.

\section{Synthesis of 4-acetamido phenoxy acetyl hydrazide (2) ${ }^{10}$}

The ethyl-4-acetamido phenoxy acetate $(0.01 \mathrm{~mol})$, hydrazine hydrate $(0.01 \mathrm{~mol})$ was refluxed in the presence of ethanol for $5 \mathrm{~h}$. The reaction mixture was cooled and then poured in to crushed ice. The solid product formed, was filtered, dried and recrystallized using ethanol.

\section{General procedure for compounds (G1-G12) ${ }^{11}$}

A mixture of 4-Acetamido phenoxy acetyl hydrazide $(0.01 \mathrm{~mol})$ and various aromatic acids $(0.01 \mathrm{~mol})$ in phosphorus oxychloride $(10 \mathrm{~mL})$ was exposed to microwaves at $350 \mathrm{~W}$, intermittently at $2 \mathrm{~min}$. intervals for $8-15 \mathrm{~min}$. Completion of the reaction process was monitored by TLC. The contents were cooled and poured into the crushed ice. It was neutralized with sodium bicarbonate solution and the solid product obtained was filtered, dried and recrystallized using ethanol.

\section{4-((5-(3-Aminophenyl)-1,3,4-oxadiazol-2-yl)methoxy)phenyl) acetamide (G1)}

Recrystallized from ethanol, (yield 54\%), mp $116{ }^{0} \mathrm{C}$. IR (KBr): $3393.16 \mathrm{~cm}^{-1}$ (Ar-NH), $1633.67 \mathrm{~cm}^{-1}(\mathrm{C}=\mathrm{N}), 1575.88 \mathrm{~cm}^{-1}(\mathrm{C}=\mathrm{C}), 1069.05 \mathrm{~cm}^{-1}(-\mathrm{C}-\mathrm{O}-\mathrm{C}-), 3132.54 \mathrm{~cm}^{-1}$ (Ar-CH). ${ }^{1} \mathrm{H}-\mathrm{NMR}: \delta 8.05(\mathrm{~s}, 1 \mathrm{H},-\mathrm{NH}), 6.45-7.4(\mathrm{~m}, 8 \mathrm{H}, \mathrm{Ar}-\mathrm{H}), 5.17\left(\mathrm{~s}, 2 \mathrm{H},-\mathrm{CH}_{2}\right), 4.10(\mathrm{~s}, 2 \mathrm{H}$, $\left.-\mathrm{NH}_{2}\right), 2.05\left(\mathrm{~s}, 3 \mathrm{H},-\mathrm{CH}_{3}\right), \mathrm{MS}: \mathrm{m} / \mathrm{z} 324\left(\mathrm{M}^{+}\right)$other peak observed at 310, 296, 282, 272, 262, 248 and 58(B).

\section{4-((5-(2-Aminophenyl)-1,3,4-oxadiazol-2-yl)methoxy)phenyl)acetamide (G2)}

Recrystallized from ethanol, (yield 56\%), mp $123{ }^{\circ} \mathrm{C}$. IR (KBr): $3381.92 \mathrm{~cm}^{-1}(\mathrm{Ar}-\mathrm{NH})$, $1677.76 \mathrm{~cm}^{-1}(\mathrm{C}=\mathrm{N}), 1530.51 \mathrm{~cm}^{-1} \quad(\mathrm{C}=\mathrm{C}), 1089.81 \mathrm{~cm}^{-1} \quad(-\mathrm{C}-\mathrm{O}-\mathrm{C}-), 3118.42 \mathrm{~cm}^{-1}$ $(\mathrm{Ar}-\mathrm{CH}) .{ }^{1} \mathrm{H}-\mathrm{NMR}: \delta 7.95(\mathrm{~s}, 1 \mathrm{H},-\mathrm{NH}), 6.46-7.45(\mathrm{~m}, 8 \mathrm{H}, \mathrm{Ar}-\mathrm{H}), 5.21\left(\mathrm{~s}, 2 \mathrm{H},-\mathrm{CH}_{2}\right), 4.05$ (s, 2H, $\left.-\mathrm{NH}_{2}\right), 2.12\left(\mathrm{~s}, 3 \mathrm{H},-\mathrm{CH}_{3}\right) . \mathrm{MS}: \mathrm{m} / \mathrm{z} 324\left(\mathrm{M}^{+}\right)$, other peak observed at 284, 262, 284, 216, 174, 156 and 80(B).

\section{4-((5-(2-Chloro-5-nitrophenyl)-1,3,4-oxadiazol-2-yl)methoxy)phenyl)acetamide (G3)}

Recrystallized from ethanol, (yield 60\%), mp $135{ }^{\circ} \mathrm{C}$. IR (KBr): $3381.95 \mathrm{~cm}^{-1}(\mathrm{Ar}-\mathrm{NH})$, $1684.44 \mathrm{~cm}^{-1}(\mathrm{C}=\mathrm{N}), 1586.2 \mathrm{~cm}^{-1}(\mathrm{C}=\mathrm{C}), 1064.25 \mathrm{~cm}^{-1}(-\mathrm{C}-\mathrm{O}-\mathrm{C}-), 1365.57 \mathrm{~cm}^{-1}(\mathrm{~N}=\mathrm{O})$, $801.35 \mathrm{~cm}^{-1}$ (C-Cl), $3130.43 \mathrm{~cm}^{-1}$ (Ar-CH). ${ }^{1} \mathrm{H}-\mathrm{NMR}: \delta 8.16(\mathrm{~s}, 1 \mathrm{H},-\mathrm{NH}), 6.74-8.36(\mathrm{~m}, 8 \mathrm{H}$, $\mathrm{Ar}-\mathrm{CH}), 5.31\left(\mathrm{~s}, 2 \mathrm{H},-\mathrm{CH}_{2}\right), 2.31\left(\mathrm{~s}, 3 \mathrm{H},-\mathrm{CH}_{3}\right) . \mathrm{MS}: \mathrm{m} / \mathrm{z} 390\left(\mathrm{M}^{+}\right)$other peak observed at $388,362,233,217,182,96$ and 78 (B). 
4-((5-(4-Chloro-3-nitrophenyl)-1,3,4-oxadiazol-2-yl)methoxy)phenyl)acetamide (G4) Recrystallized from ethanol, (yield 62\%), mp $203{ }^{\circ} \mathrm{C}$. IR (KBr): $3382.02 \mathrm{~cm}^{-1}$ (Ar-NH), $1677.79 \mathrm{~cm}^{-1}(\mathrm{C}=\mathrm{N}), 1530.6 \mathrm{~cm}^{-1}(\mathrm{C}=\mathrm{C}), 1089.68 \mathrm{~cm}^{-1}(-\mathrm{C}-\mathrm{O}-\mathrm{C}-), 1372.45 \mathrm{~cm}^{-1}(\mathrm{~N}=\mathrm{O})$, $813.038 \mathrm{~cm}^{-1}(\mathrm{C}-\mathrm{Cl}), 3117.5 \mathrm{~cm}^{-1}$ (Ar-CH). ${ }^{1} \mathrm{H}-\mathrm{NMR}: \delta 8.24(\mathrm{~s}, 1 \mathrm{H},-\mathrm{NH}), 6.83-8.12(\mathrm{~m}, 8 \mathrm{H}$, Ar- $\mathrm{CH}$ ), 5.35(s, $\left.2 \mathrm{H},-\mathrm{CH}_{2}\right), 2.07\left(\mathrm{~s}, 3 \mathrm{H},-\mathrm{CH}_{3}\right)$. MS: $\mathrm{m} / \mathrm{z} 390\left(\mathrm{M}^{+}\right)$other peak observed at $388,380,261,182,167,156$ and $81(\mathrm{~B})$.

\section{4-((5-(2-Fluorophenyl)-1,3,4-oxadiazol-2-yl)methoxy)phenyl)acetamide (G5)}

Recrystallized from ethanol, (yield 64\%), mp $187^{\circ} \mathrm{C}$. IR (KBr): $3392.09 \mathrm{~cm}^{-1}$ (Ar-NH), 1617.53 $\mathrm{cm}^{-1}(\mathrm{C}=\mathrm{N}), 1528.16 \mathrm{~cm}^{-1}(\mathrm{C}=\mathrm{C}), 1093.52 \mathrm{~cm}^{-1}$ (-C-O-C-), $1371.78 \mathrm{~cm}^{-1}(\mathrm{C}-\mathrm{F}), 3114.61 \mathrm{~cm}^{-1}$ (Ar-CH). ${ }^{1} \mathrm{H}-\mathrm{NMR}: 8.09(\mathrm{~s}, 1 \mathrm{H},-\mathrm{NH}), 6.7-8.01(\mathrm{~m}, 8 \mathrm{H}, \mathrm{Ar}-\mathrm{CH}), 5.21\left(\mathrm{~s}, 2 \mathrm{H},-\mathrm{CH}_{2}\right), \delta 2.21(\mathrm{~s}, 3 \mathrm{H}$, $\left.-\mathrm{CH}_{3}\right)$. MS: $\mathrm{m} / \mathrm{z} 327\left(\mathrm{M}^{+}\right)$other peak observed at 310, 299, 282, 266, 249, 232, 104 and 75(B).

\section{4-((5-(4-Methoxyphenyl)-1,3,4-oxadiazol-2-yl)methoxy)phenyl)acetamide (G6)}

Recrystallized from ethanol, (yield 56\%), mp $174{ }^{\circ} \mathrm{C}$. IR ( $\mathrm{KBr}$ ): $3457.55 \mathrm{~cm}^{-1}(\mathrm{Ar}-\mathrm{NH})$, $1625.05 \mathrm{~cm}^{-1}(\mathrm{C}=\mathrm{N}), 1585.42 \mathrm{~cm}^{-1}(\mathrm{C}=\mathrm{C}), 1094.6 \mathrm{~cm}^{-1}(-\mathrm{C}-\mathrm{O}-\mathrm{C}-), 3123.84 \mathrm{~cm}^{-1}(\mathrm{Ar}-\mathrm{CH})$. ${ }^{1} \mathrm{H}-\mathrm{NMR}: \delta 8.14(\mathrm{~s}, 1 \mathrm{H}, \mathrm{NH}) .6 .8-7.56(\mathrm{~m}, 8 \mathrm{H}, \mathrm{Ar}-\mathrm{CH}), 2.22\left(\mathrm{~s}, 3 \mathrm{H},-\mathrm{CH}_{3}\right), 3.70(\mathrm{~s}, 3 \mathrm{H}$, $\left.-\mathrm{CH}_{3}\right), 5.21\left(\mathrm{~s}, 2 \mathrm{H},-\mathrm{CH}_{2}\right), 8.14(\mathrm{~s}, 1 \mathrm{H}, \mathrm{NH})$. MS: $\mathrm{m} / \mathrm{z} 339\left(\mathrm{M}^{+}\right)$other peak observed at 324, $307,296,249,160,132,116$ and 87(B).

\section{4-((5-(4-Ethoxyphenyl)-1,3,4-oxadiazol-2-yl)methoxy)phenyl)acetamide (G7)}

Recrystallized from ethanol, (yield 64\%), mp $175^{\circ} \mathrm{C}$. IR (KBr): $3430.18 \mathrm{~cm}^{-1}$ (Ar-NH), $1630.28 \mathrm{~cm}^{-}$ ${ }^{1}(\mathrm{C}=\mathrm{N}), 1589.85 \mathrm{~cm}^{-1}(\mathrm{C}=\mathrm{C}), 1091.04 \mathrm{~cm}^{-1}$ (-C-O-C-), $3123.31 \mathrm{~cm}^{-1}$ (Ar-CH). ${ }^{1} \mathrm{H}-\mathrm{NMR}$ : $\delta 8.07(\mathrm{~s}, 1 \mathrm{H},-\mathrm{NH}), 6.74-7.37(\mathrm{~m}, 8 \mathrm{H}, \mathrm{Ar}-\mathrm{CH}), 5.24\left(\mathrm{~s}, 2 \mathrm{H},-\mathrm{CH}_{2}\right), 2.25\left(\mathrm{~s}, 3 \mathrm{H},-\mathrm{CH}_{3}\right), 1.31(\mathrm{~s}, 3 \mathrm{H}$, $-\mathrm{CH}_{3}$ ). MS: $\mathrm{m} / \mathrm{z} 353\left(\mathrm{M}^{+}\right)$other peak observed at $335,325,302,249,204,183,160,115$, and $91(\mathrm{~B})$.

\section{4-((5-o-Tolyl-1,3,4-oxadiazol-2-yl)methoxy)phenyl)acetamide (G8)}

Recrystallized from ethanol, (yield 63\%), mp $211{ }^{\circ} \mathrm{C}$. IR ( $\mathrm{KBr}$ ): $3354.23 \mathrm{~cm}^{-1}(\mathrm{Ar}-\mathrm{NH})$, $1632.99 \mathrm{~cm}^{-1}(\mathrm{C}=\mathrm{N}), 1584.68 \mathrm{~cm}^{-1}(\mathrm{C}=\mathrm{C}), 1092.53 \mathrm{~cm}^{-1}(-\mathrm{C}-\mathrm{O}-\mathrm{C}-), 3123.84 \mathrm{~cm}^{-1}$ (Ar-CH). ${ }^{1} \mathrm{H}-\mathrm{NMR}: \delta 8.16(\mathrm{~s}, 1 \mathrm{H},-\mathrm{NH}), 7.41(\mathrm{~m}, 8 \mathrm{H}, \mathrm{Ar}-\mathrm{CH}), 2.31\left(\mathrm{~s}, 3 \mathrm{H},-\mathrm{CH}_{3}\right), 5.31\left(\mathrm{~s}, 2 \mathrm{H},-\mathrm{CH}_{2}\right)$. MS: $\mathrm{m} / \mathrm{z} 323\left(\mathrm{M}^{+}\right)$other peak observed at 277, 266, 249, 232, 158, 140, 132 and 80(B).

\section{4-((5-(3-Nitrophenyl)-1,3,4-oxadiazol-2-yl)methoxy)phenyl)acetamide (G9)}

Recrystallized from ethanol, (yield 64\%), mp $169^{\circ} \mathrm{C}$. IR (KBr): $3382.43 \mathrm{~cm}^{-1}(\mathrm{Ar}-\mathrm{NH}), 1703.01 \mathrm{~cm}^{-1}$ $(\mathrm{C}=\mathrm{N}), 1592.32 \mathrm{~cm}^{-1}(\mathrm{C}=\mathrm{C}), 1088.54 \mathrm{~cm}^{-1}($ (-C-O-C- $), 1378.11 \mathrm{~cm}^{-1}(\mathrm{~N}=\mathrm{O}), 3112.69 \mathrm{~cm}^{-1}$ (Ar-CH). ${ }^{1} \mathrm{H}-\mathrm{NMR}: \delta 8.13(\mathrm{~s}, 1 \mathrm{H},-\mathrm{NH}), 6.41-7.8(\mathrm{~m}, 8 \mathrm{H}, \mathrm{Ar}-\mathrm{CH}), 2.42\left(\mathrm{~s}, 3 \mathrm{H},-\mathrm{CH}_{3}\right), 5.21(\mathrm{~s}, 2 \mathrm{H}$, $\mathrm{CH}_{2}$ ). MS: $\mathrm{m} / \mathrm{z} 354\left(\mathrm{M}^{\dagger}\right)$ other peak observed at 335, 302, 287, 249, 226, 204, 127, 103 and 89(B).

\section{4-((5-(2,6-Difluorophenyl)-1,3,4-oxadiazol-2-yl)methoxy)phenyl)acetamide (G10)}

Recrystallized from ethanol, (yield 53\%), mp $180{ }^{\circ} \mathrm{C}$. IR (KBr): $3368.58 \mathrm{~cm}^{-1}$ (Ar-NH), 1673.42 $\mathrm{cm}^{-1}(\mathrm{C}=\mathrm{N}), 1545.03 \mathrm{~cm}^{-1}(\mathrm{C}=\mathrm{C}), 1085.04 \mathrm{~cm}^{-1}$ (-C-O-C-), $1378.04 \mathrm{~cm}^{-1}(\mathrm{C}-\mathrm{F}), 3115.62 \mathrm{~cm}^{-1}$ (Ar-CH). ${ }^{1} \mathrm{H}-\mathrm{NMR}: \delta 8.03(\mathrm{~s}, 1 \mathrm{H},-\mathrm{NH}), 6.6-7.82(\mathrm{~m}, 8 \mathrm{H}, \mathrm{Ar}-\mathrm{CH}), 2.5\left(\mathrm{~s}, 3 \mathrm{H},-\mathrm{CH}_{3}\right), 5.22(\mathrm{~s}, 2 \mathrm{H}$, $\left.-\mathrm{CH}_{2}\right)$. MS m/z $345\left(\mathrm{M}^{+}\right)$other peak observed at 333, 325, 286, 183, 160, 140, 115 and 95(B).

4-((5-(2-Hydroxyphenyl)-1,3,4-oxadiazol-2-yl)methoxy)phenyl)acetamide (G11) Recrystallized from ethanol, (yield 68\%), mp $215{ }^{\circ} \mathrm{C}$. IR (KBr): $3382.83 \mathrm{~cm}^{-1}(\mathrm{Ar}-\mathrm{NH})$, $1654.42 \mathrm{~cm}^{-1}(\mathrm{C}=\mathrm{N}), 1541.89 \mathrm{~cm}^{-1}(\mathrm{C}=\mathrm{C}), 1090.01 \mathrm{~cm}^{-1}$ (-C-O-C-), $3118.84 \mathrm{~cm}^{-1}$ (Ar-CH). ${ }^{1} \mathrm{H}-\mathrm{NMR}: \delta 8.00(\mathrm{~s}, 1 \mathrm{H},-\mathrm{NH}), 6.7-7.6(\mathrm{~m}, 6 \mathrm{H}, \mathrm{Ar}-\mathrm{CH}), 2.11\left(\mathrm{~s}, 3 \mathrm{H},-\mathrm{CH}_{3}\right), 5.12\left(\mathrm{~s}, 2 \mathrm{H},-\mathrm{CH}_{2}\right)$. MS: $\mathrm{m} / \mathrm{z} 325\left(\mathrm{M}^{+}\right)$other peak observed at 318, 292, 276, 249, 236, 203, 182, 134, 116(B). 


\section{4-((5-(Pyridin-3-yl)-1,3,4-oxadiazol-2-yl)methoxy)phenyl)acetamide (G12)}

Recrystallized from ethanol, (yield 62\%), mp $210{ }^{\circ} \mathrm{C}$. IR (KBr): $3482.34 \mathrm{~cm}^{-1}$ (Ar-NH), $1654.26 \mathrm{~cm}^{-1}(\mathrm{C}=\mathrm{N}), 1528.16 \mathrm{~cm}^{-1}(\mathrm{C}=\mathrm{C}), 1093.52 \mathrm{~cm}^{-1}(-\mathrm{C}-\mathrm{O}-\mathrm{C}-), 3138.86 \mathrm{~cm}^{-1}(\mathrm{Ar}-\mathrm{CH})$. ${ }^{1} \mathrm{H}-\mathrm{NMR}: \delta 8.13(\mathrm{~s}, 1 \mathrm{H},-\mathrm{NH}), 7.31-7.82(\mathrm{~m}, 8 \mathrm{H}, \mathrm{Ar}-\mathrm{CH}), 2.1\left(\mathrm{~s}, 3 \mathrm{H},-\mathrm{CH}_{3}\right), 5.2\left(\mathrm{~s}, 2 \mathrm{H},-\mathrm{CH}_{2}\right)$. MS: $m / z 310\left(\mathrm{M}^{+}\right)$other peak observed at 289, 248, 231, 157, 131, 115, 103, 96(B).

\section{Antimicrobial activity}

\section{Antibacterial and antifungal activity}

The antibacterial and antifungal activity of twelve compounds (G1-G12) was investigated by employing the paper disc diffusion method ${ }^{12}$. Representative organisms selected for evaluation of antibacterial activity were $S$. aureus, E. coli and antifungal activity were A. nigar. The antibacterial and antifungal activity of each of the compounds was evaluated in 50, 100, 150 $\mu \mathrm{g} \mathrm{mL} \mathrm{m}^{-1}$ concentrations. The compounds were tasted as a suspension in DMF (99.79\%). An important and useful control drug ciprofloxacin for antibacterial and ketoconazole for antifungal was also tested under similar conditions, compare with the results.

The result indicates that all of the synthesized compounds showed moderate to strong activity against bacterial and fungal strains. Compounds G2, G5, G7 and G10 were found to be exhibit good antibacterial activity against S.aureus, E.coli and Compounds G3, G5, G7 and G9 exhibit antifungal activity against A.nigar. From above observation it is prove that the 2,5-disubstituted 1,3,4-oxadiazole derivatives are active and also the substituents amino, fluoro, ethoxy and nitro in 2,5-disubstituted 1,3,4-oxadiazole derivatives plays a important role in the antimicrobial screening. The evaluation of antibacterial and antifungal activity of the synthesized compounds is shown in Table 1.

Table 1. Evaluation of antimicrobial activity of the compounds

\begin{tabular}{|c|c|c|c|c|c|c|c|c|c|}
\hline \multirow{4}{*}{ Compounds } & \multicolumn{6}{|c|}{ Bacteria } & \multicolumn{3}{|c|}{ Fungi } \\
\hline & \multicolumn{3}{|c|}{ S.aureus } & \multicolumn{3}{|c|}{ E.coli } & \multicolumn{3}{|c|}{ A.nigar } \\
\hline & \multicolumn{9}{|c|}{ Concentration, $\mu \mathrm{g} / \mathrm{mL}$} \\
\hline & $50 \mathrm{mg}$ & $100 \mathrm{mg}$ & $150 \mathrm{mg}$ & $50 \mathrm{mg}$ & $100 \mathrm{mg}$ & $150 \mathrm{mg}$ & $50 \mathrm{mg}$ & $100 \mathrm{mg}$ & $150 \mathrm{mg}$ \\
\hline G1 & 18 & $21(36)$ & 25 & 19 & $21(31)$ & 25 & 19 & $21(38)$ & 24 \\
\hline G2 & 22 & $24(15)$ & 29 & 22 & $25(20)$ & 29 & 20 & $22(29)$ & 23 \\
\hline G3 & 23 & $27(33)$ & 31 & 18 & $21(35)$ & 24 & 23 & $25(19)$ & 28 \\
\hline G4 & 17 & $19(38)$ & 23 & 15 & $18(40)$ & 20 & 19 & $21(33)$ & 28 \\
\hline G5 & 25 & $29(17)$ & 32 & 23 & $27(18)$ & 29 & 22 & $25(20)$ & 31 \\
\hline G6 & 20 & $24(34)$ & 27 & 19 & $21(37)$ & 24 & 18 & $21(25)$ & 24 \\
\hline G7 & 23 & $25(20)$ & 27 & 24 & $28(21)$ & 31 & 23 & $26(17)$ & 31 \\
\hline G8 & 20 & $24(35)$ & 28 & 18 & $21(35)$ & 25 & 20 & $24(40)$ & 27 \\
\hline G9 & 17 & $19(31)$ & 22 & 19 & $22(33)$ & 24 & 22 & $25(23)$ & 29 \\
\hline G10 & 24 & $28(16)$ & 32 & 23 & $27(19)$ & 31 & 20 & $21(25)$ & 24 \\
\hline G11 & 19 & $22(25)$ & 27 & 19 & $22(22)$ & 24 & 19 & $22(35)$ & 25 \\
\hline G12 & 16 & $20(39)$ & 25 & 21 & $24(29)$ & 26 & 23 & $25(32)$ & 27 \\
\hline $\begin{array}{c}\text { Ciprofloxacin, } \\
100 \mu \mathrm{g} / \mathrm{mL}\end{array}$ & \multicolumn{3}{|c|}{$38(0.2)$} & \multicolumn{3}{|c|}{$38(0.3)$} & \multicolumn{3}{|c|}{-} \\
\hline Ketoconazole, & \multirow{2}{*}{\multicolumn{3}{|c|}{-}} & \multirow{2}{*}{\multicolumn{3}{|c|}{-}} & \multirow{2}{*}{\multicolumn{3}{|c|}{$38(6.1)$}} \\
\hline $\begin{array}{c}100 \mu \mathrm{g} / \mathrm{mL} \\
\text { Control }\end{array}$ & & & & & & & & & \\
\hline (DMF) & \multicolumn{3}{|c|}{ - } & \multicolumn{3}{|c|}{-} & \multicolumn{3}{|c|}{ - } \\
\hline
\end{tabular}




\section{Determination of MIC}

\section{Agar streak dilution method ${ }^{13}$}

MIC of the synthesized compounds was determined by agar streak dilution method. A stock solution of the synthesized compounds $(100 \mu \mathrm{g} / \mathrm{mL})$ in dimethyl formamide was prepared and graded quantities of the test compounds were incorporated in specified quantities of molten nutrient agar medium. A specified quantity of the medium containing the compounds was poured into a petri dish to give a depth of 3-4 $\mathrm{mm}$ and allowed to solidify. Suspension of the microorganism were prepared to contain approximately $10^{5} \mathrm{cfu} \mathrm{m} / 1$ and applied to plates with serially diluted compounds in dimethyl formamide to be tested and incubated at $37{ }^{\circ} \mathrm{C}$ for $24 \mathrm{~h}$ for bacteria and fungi. The MIC was considered to be the lowest concentration of the test substance exhibiting no visible growth of bacteria on the plate. The observed MIC is represented in Table 1.

\section{Results and Discussion}

All the compounds of this report have been screened for their antimicrobial activity. The perusal of the results (Table 1) indicates that the range of inhibition zones of tested at different concentration of 50, 100 and $150 \mu \mathrm{g} / \mathrm{mL}$. Compounds G2, G5, G7 and G10 were found to be exhibit promising antibacterial activity against S.aureus and E.coli and compounds G3, G5, G7 and G9 were found to be exhibit good antifungal activity against A.nigar. The compounds (G5) found to be exhibit highest antibacterial activity against S.aureus (MIC: $17 \mu \mathrm{g} / \mathrm{mL}$ ), E.coli (MIC $18 \mu \mathrm{g} / \mathrm{mL}$ ), compound (G7) found to be exhibit antifungal activity against A.nigar (MIC: $17 \mu \mathrm{g} / \mathrm{mL}$ ). They showed different effects on antibacterial and antifungal species. The IR, Mass and NMR spectral data confirmed their molecular structure.
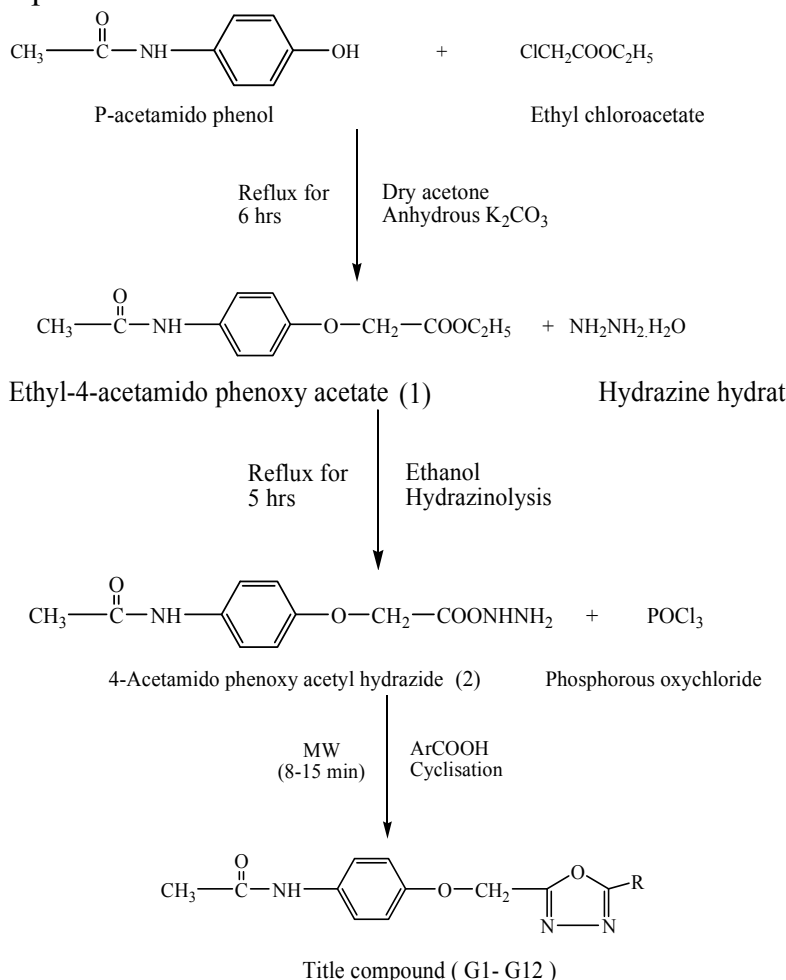

Scheme 1. Synthesis of 2,5-disubstituted 1,3,4-oxadiazole 


\section{Conclusion}

We have described an easy preparation procedure of 2, 5-disubstituted 1,3,4-oxadiazole derivatives from $p$-acetamido phenol, ethyl chloroacetate, hydrazine hydrate, phosphorous oxychloride and different benzoic acids and its antimicrobial activity. All these compounds containing 1,3,4-oxadiazole moiety is more active and plays an important role in antimicrobial activity. The structures of all the compounds were confirmed by IR, NMR and MS spectral data.

\section{Acknowledgment}

The authors thank to the management and the principal, Department of Pharmaceutical Chemistry, C.L.Baid Metha College of Pharmacy, Thoraipakkam, Chennai for providing laboratory facilities. The authors also thanks to Dr. Ceeal Analytical Lab, Chennai for IR and IIT Chennai, for NMR and Mass spectral analysis.

\section{References}

1. Chawla R, Sharma P C, Arora A, Parameswaran M K, Michael S and Ravi T K, Acta Pol Pharma- Drug Res., 2010, 67(3), 247-253.

2. Zarghi A, Faizi M, Shafaghi B, Ahadian A, Khojastehpoor H R, Zanganeh V and Sayyed A, Bioorg Med Chem Lett., 2005, 15(12), 3126-3129.

3. Husain M.I and Shukla M K, J Indian Chem Soc., 1978, 55, 826-828.

4. Shivananda W, Suchethakumari N and Vasudeva A, Indian J Chem., 2008, 47B, 439-448.

5. Cao S, Qian X, Song G and Huang Q, J Fluorine Chem., 2002, 117, 63-66.

6. Rajak H, Kharya M D and Mishra P, Arch Pharm., 2008, 341(4), 247-261.

7. Adelstein G W, Yen C H, Dajani E Z and Bianchi R G, J Med Chem., 1976, 19(10), 1221-1225.

8. Aboraia A.S, Abdel-Rahman H.M, Mahfouz N.M and E.L-Gendy M.A, Bioorg Med Chem., 2006, 14, 1236-1246.

9. Girish R. Bankar, K. Nandakumar, Pawan G. Nayak, Anjali Thakur, M. Rao Chamallamudi, Gopalan Kutty Nampurath, Chemico-Biological Interactions., 2009, 181(13), 377-382.

10. Ilango K, Valentina P, Umarani N and Kumar T., Indian J Chem., 2009, 1(1), 72-78.

11. Khan K M, Rani M and Haider S M, Lett Org Chem., 2004, 1, 151.

12. Varma R S, Imran S A and Nobles W L, J Pharm Sci., 1973, 62, 140.

13. Hawkey P M and Lewis D A, Medical bacteriology- A Practical Approach. United Kingdom: Oxford University Press, 1994, 181-94. 


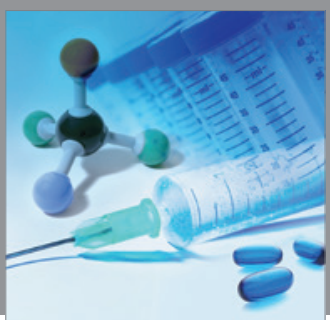

International Journal of

Medicinal Chemistry

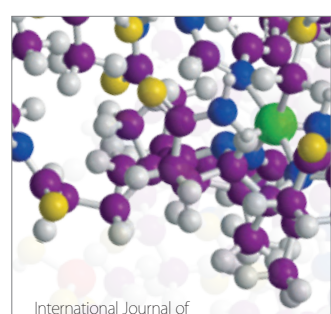

Carbohydrate Chemistry

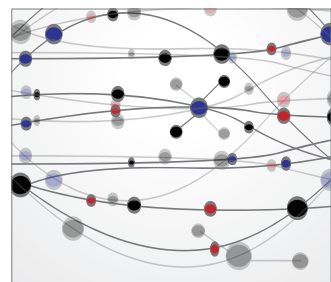

The Scientific World Journal
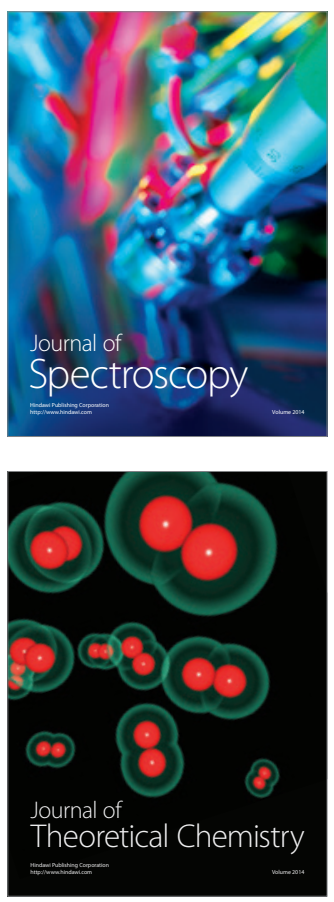
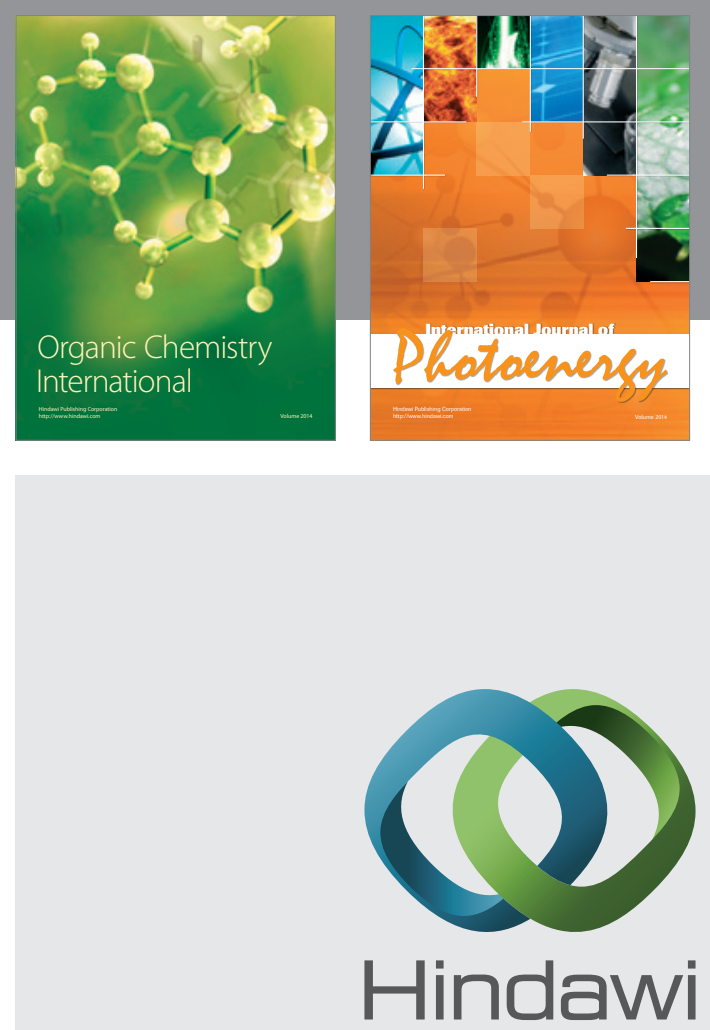

Submit your manuscripts at

http://www.hindawi.com
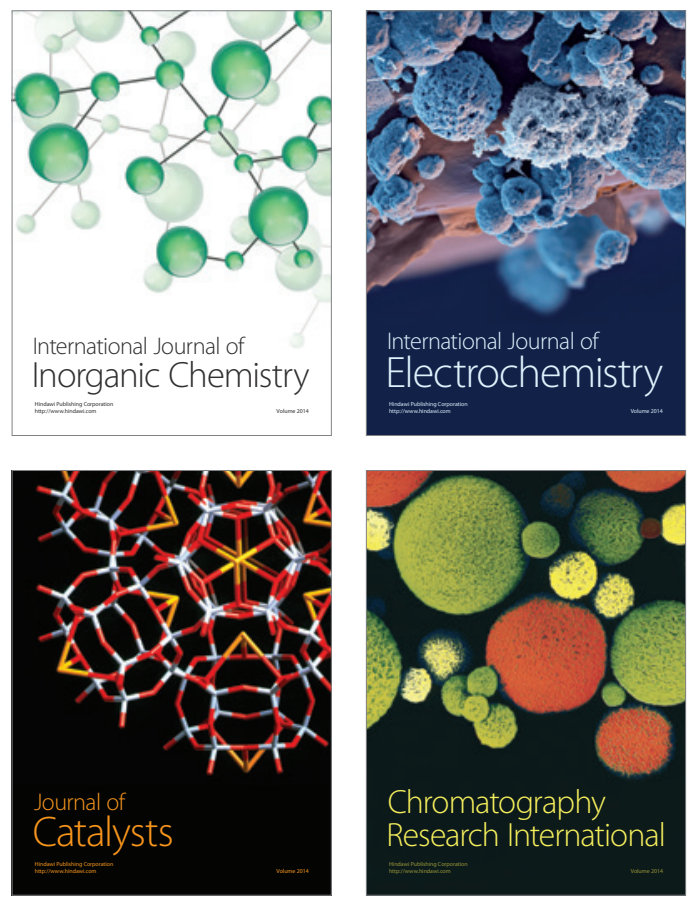
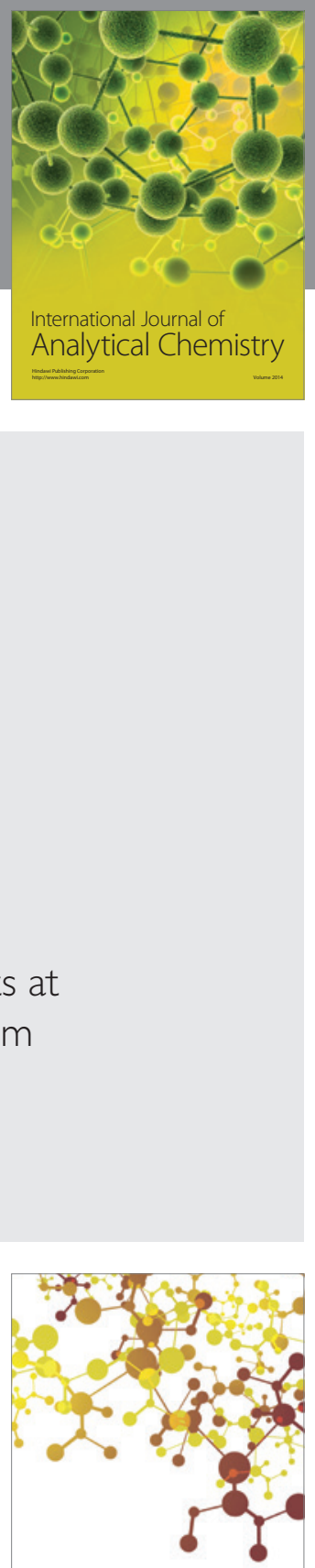

Journal of

Applied Chemistry
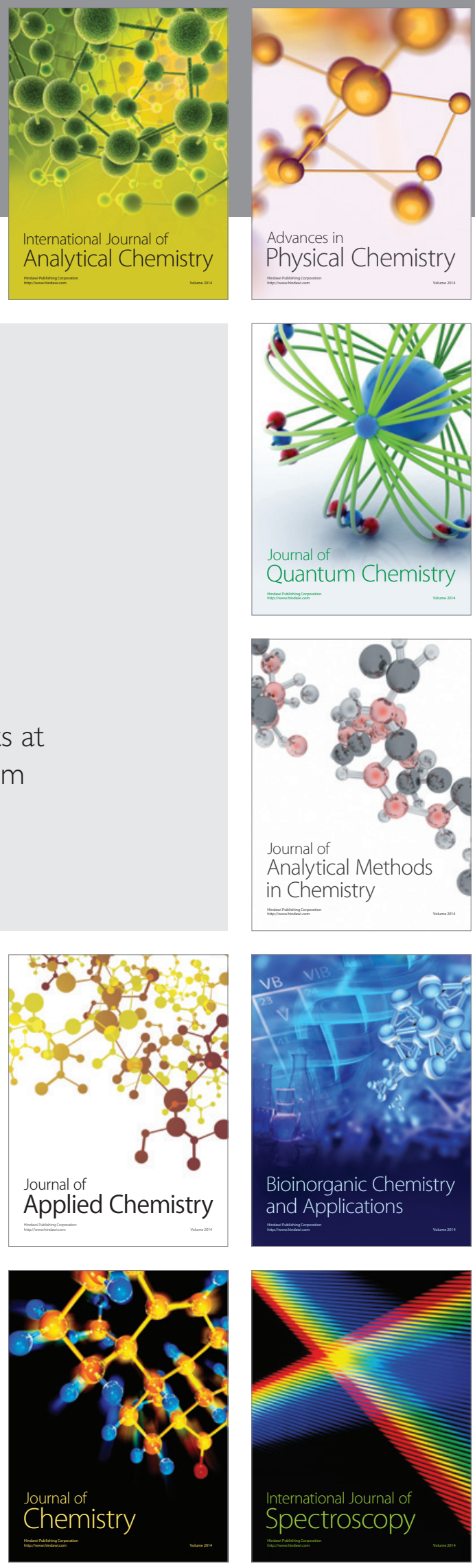